\title{
The importance of a correct dead time setting in isotope ratio mass spectrometry: Implementation of an electronically determined dead time to reduce measurement uncertainty
}

\author{
Ulrika Nygren $^{\mathrm{a}, \mathrm{b}, *}$, Henrik Ramebäck ${ }^{\mathrm{a}}$, Michael Berglund ${ }^{\mathrm{c}}$, Douglas C. Baxter ${ }^{\mathrm{d}}$ \\ ${ }^{a}$ Swedish Defence Research Agency (FOI), Division of NBC Analysis, SE-901 82 Umeå, Sweden \\ ${ }^{\mathrm{b}}$ Division of Chemistry, Luleå University of Technology, SE-971 87 Luleå, Sweden \\ ${ }^{\mathrm{c}}$ Institute for Reference Materials and Measurements, EC-JRC-IRMM, B-2440 Geel, Belgium \\ ${ }^{d}$ Analytica AB, Aurorum 10, SE-977 75 Luleå, Sweden
}

Received 15 March 2006; received in revised form 11 May 2006; accepted 15 May 2006

Available online 11 July 2006

\begin{abstract}
Dead time determinations on a mass spectrometry system with ion counting detection can either be done using an isotope ratio measurement approach or via electronic examination of individual components, e.g., the pulse amplifier. Depending on the dead time of each component in the signal chain, the electronically determined result may not represent the true value for the total system, i.e., there might be a series of hardware related dead times. However, a hardware set artificial dead time in the pulse counting system that is long enough might yield a system dead time that can be represented by this set dead time solely. It is shown in this work that a carefully chosen, artificially introduced set dead time yields a system that may be characterized to a much lower uncertainty than is possible using ratio-based measurement approaches. Ultimately, the impact of the dead time on the combined uncertainty of an isotope abundance ratio measurement may be negligible even at very high count rates, i.e., $>1$ Mcps. In other words, incorporating the dead time contribution in a combined uncertainty calculation would no longer be necessary.

(C) 2006 Elsevier B.V. All rights reserved.
\end{abstract}

Keywords: Dead time; Mass spectrometry; Isotope ratio; Uncertanty contribution; Pulse counting

\section{Introduction}

In any pulse counting system there is a specific time interval after which no new events can be counted. This interval is defined as the dead time. The dead time of the detection system is one of several factors that may contribute to bias as well as to uncertainty in isotope abundance ratio measurements using mass spectrometers in counting mode. An accurate and precise determination of the dead time is therefore required. While an error in the value used for dead time correction will lead to bias in an isotope ratio measurement, any uncertainty will contribute to the combined uncertainty in the result. The effect on the combined uncertainty of a ratio measurement will depend on both the magnitude of the dead time uncertainty and the difference in intensities between the signals forming the ratio to be mea-

\footnotetext{
* Corresponding author. Tel.: +4690 106754; fax: +46 90106803.

E-mail address: ulrika.nygren@ foi.se (U. Nygren).
}

sured. Basically two approaches can be adopted to minimize the contribution of the dead time uncertainty; one is to suppress the signals in order to reduce the effect of the dead time [1], the other is to determine the dead time with a higher precision.

Ratio-based methods are usually applied for dead time determination in inductively coupled plasma mass spectrometry (ICP-MS). By observing the change in signal ratio upon an intensity change, the dead time of the detection system can be determined. Several different approaches for evaluating the dead time based on isotope ratio measurements have been reported [2-6]. However, one limitation of this method is the precision with which the dead time can be determined. Nelms et al. [6] reported uncertainties in the range of 4-20 ns $(k=1)$ for different methods, and Appelblad and Baxter [4] reported uncertainties down to about $1 \mathrm{~ns}(k=1)$ at best. If the dead time instead is determined via electronic measurement [5], the precision is mainly governed by the sampling rate of the measuring device. This is a relatively easy approach to reduce dead time contribution to the overall uncertainty in mass 
spectrometric isotope ratio determinations that has not yet been described.

However, an electronically determined value may not represent the total counting system, but rather, only the dead time of the component measured, e.g., the pulse amplifier. In addition, different parts of a signal chain each have their own dead time, e.g., the electron multiplier and the pulse amplifier are each characterized by their individual values. In the case of the electron multiplier, its dead time may be difficult to determine, and it may also only be approximately constant [7]. In the case of the pulse amplifier, a well-defined artificial dead time may be implemented electronically. One reason for this is that, if long enough compared to the dead time of the electron multiplier, it will represent the dead time of the total system [8]. Furthermore, a well known, although large, correction is preferable to a small but uncertain one [7].

It is also possible to implement different types of dead times in an electronic circuit [8], the two types considered being termed paralyzable and non-paralyzable [9]. The difference between the two types is that, in the paralyzable case, a new dead time is extended by additional events, i.e., the irresponsive period is not fixed in duration. A non-paralyzable detection system is in general preferred, since the paralyzable system may, at very high count rates, show apparently low readings [9]. An artificial dead time is also introduced in order to mask afterpulses that occur promptly after a main event in the detector $[5,10]$.

The common model used for dead time correction in both nuclear spectroscopy and mass spectrometry using ion counting detection is [9]

$I_{\mathrm{t}}=\frac{I_{\mathrm{m}}}{1-I_{\mathrm{m}} \tau}$

where $I_{\mathrm{t}}$ is the corrected intensity, $I_{\mathrm{m}}$ the observed intensity and $\tau$ the dead time. This model applies only for the non-paralyzable dead time type and for a Poissonian process at the detector $[8,9]$.

In this work, the pulse counting system of a sector field ICP-MS instrument was studied by means of dead time measurements. Dead times were determined both electronically and with ratio-based methods using the certified reference materials IRMM 073/3, 5 and 7. The 073-series has two certified uranium ratios. First, $R_{235 / 238}$ can be used for an inherent mass bias correction since this isotope ratio is close to unity and will not be affected by a dead time. The other isotope ratio, $R_{233 / 238}$, is varied over almost six orders of magnitude and can, with the exception of $073 / 1$ for which $R_{233 / 238} \approx 1$, be used for dead time determination. Furthermore, since a circuit with three different possible dead time settings is implemented in the pulse amplifier of the instrument used in this study, all three settings were examined. In this paper the consequences of using too low dead time setting are outlined, and some recommendations are made.

\section{Materials and methods}

IRMM 073/3, 5 and 7 materials were used for the ratio-based dead time measurements. Sub-boiling distilled nitric acid and water from a Milli-Q Element system (Millipore, Molsheim, France) was used for all preparation and dilution of these materi- als, these operations being carried out in a class 1000 clean-room in order to avoid contamination by natural uranium.

All ICP-MS measurements were performed using an Element2 (Thermo-Finnigan, Bremen, Germany). The instrument was equipped with a semi-demountable quartz Fassel torch and a CD-2 Guard Electrode. Sample introduction was performed using a conical nebulizer (GlassExpansion, Melbourne, Australia) and a cyclonic spray chamber ("Twister", GlassExpansion). Self-aspiration, resulting in a sample uptake rate of approximately $0.2 \mathrm{ml} \mathrm{min}^{-1}$, was used throughout the investigation. The guard electrode was grounded and the software induced dead time correction inactivated. Torch position, lens parameters and the nebulizer gas flow rate were optimised daily to obtain maximum sensitivity for ${ }^{238} \mathrm{U}^{+}$. All measurements were performed using low resolution $(m / \Delta m=300)$ with the instrument and data acquisition settings shown in Table 1.

Electronic dead time measurements were made using a Gage CompuScope 82G (Gage Applied Technologies Inc., Lachine, Canada), plugged in to the $\mathrm{J} 2801$ contact of the pulse amplifier board. A solution containing natural uranium of about $1 \mu \mathrm{gl}^{-1}$ was used, and the magnet mass was set constant to $m / z=238$. Waveforms were sampled for about $2 \mathrm{~h}$ at a scope sampling rate of $2 \mathrm{GHz}$. The minimum pulse-to-pulse time then represents the dead time, i.e., the time between the trigger pulse and the next nearest visible pulse on the scope. The CompuScope was also used to measure pulses directly from the electron multiplier. It was then plugged in to the contact from the electron multiplier located on the housing of the electrostatic analyzer (ESA).

The pulse amplifier (part no. 2030200) originally delivered with the Element 2 was factory fixed at the lowest dead time setting (about $10 \mathrm{~ns}$ ), and was therefore modified in order to facilitate manual changing of the dead time by a jumper on the electronic board. Thus, the amplifier could then be configured to different artificial dead time settings of about 10, 20 and $70 \mathrm{~ns}$. Dead time measurements with the ratio method were evaluated both according to Appelblad and Baxter [4] and Ramebäck et al. [5].

Table 1

Instrument and data acquisition settings for the ICP-SFMS measurements

\begin{tabular}{ll}
\hline Instrument settings & \\
Argon flow rates & \\
$\quad$ Cooling gas $\left(1 \mathrm{~min}^{-1}\right)$ & 14 \\
$\quad$ Auxiliary gas $\left(1 \mathrm{~min}^{-1}\right)$ & 1 \\
$\quad$ Nebulizer gas $\left(1 \mathrm{~min}^{-1}\right)$ & $\sim 0.95$ \\
RF power $(\mathrm{W})$ & 1300 \\
Sample cone & Nickel, $1.1 \mathrm{~mm}$ orifice diameter \\
Skimmer cone & Nickel, $0.8 \mathrm{~mm}$ orifice diameter \\
& \\
Data acquisition settings: & 300 \\
Resolution $(m / \Delta m)$ & Pulse counting \\
Detection mode & E-scan \\
Data acquisition mode & $233 \mathrm{U}^{+},{ }^{235} \mathrm{U}^{+},{ }^{238} \mathrm{U}^{+}$ \\
Isotopes & $5 \mathrm{~ms}\left(\mathrm{except}\right.$ for ${ }^{233} \mathrm{U}^{+}$where $10 \mathrm{~ms}$ was used) \\
Sampling time & 10 \\
Mass window $(\%)$ & 50 \\
Samples per peak & 2000 \\
Number of scans & $1 \mathrm{~ms}(\mathrm{default}$ minimum) \\
Settling time & $233.0391 \mathrm{U}$ \\
Magnet mass & \\
&
\end{tabular}


Throughout this study, the "raw data" or "channel data" that can be obtained from the instrument software were used, and evaluations of these data were performed manually. This was done due to the discovery of a post-acquisition introduced bias in the software evaluations [11]. The uncertainties in the results were calculated according to ISO/GUM [12] using the software GUM Workbench ${ }^{\circledR}$ (Metrodata GmbH, D-79639 GrenzachWyhlen, Germany). All reported uncertainties are expanded uncertainties with a coverage factor of two $(k=2)$, unless otherwise stated.

\section{Results and discussion}

Table 2 shows the results of both ratio and electronically determined dead times for all settings of the pulse amplifier. It is clearly seen that consistency between the two approaches is only reached for the longest dead time setting of the pulse amplifier, i.e., for about $70 \mathrm{~ns}$. The plausible explanation for this is that the pulse counting system at this setting can be characterized by the dead time implemented in the pulse amplifier alone. For the other settings, the pulse amplifier contribution is not long enough to mask the dead times of the other components [10]. It can also be seen from Table 2 that the uncertainties of the electronically determined dead times are lower than those of the ratio methods.

An interesting fact is that the pulse width from the electron multiplier was measured to about $16 \mathrm{~ns}$. The dead time of the electron multiplier will probably be on the order of the width of the pulses produced by this device [9]. At the lowest dead time setting on the amplifier board the ratio method probably determines a value dominated by the electron multiplier. Overlapping pulses were observed from the electron multiplier, which indicates paralyzable behavior.

The ratio determined dead times were evaluated using two methods reported by Appelblad and Baxter [4] and Ramebäck et al. [5]. In principal, both methods are based on regression analysis for the determination of $\tau$. The difference between the methods is that in the Appelblad and Baxter approach, the mass bias is also evaluated from the regression analysis while the method used by Ramebäck et al. is based on corrections for mass bias in each measuring point. In Table 2, all individual

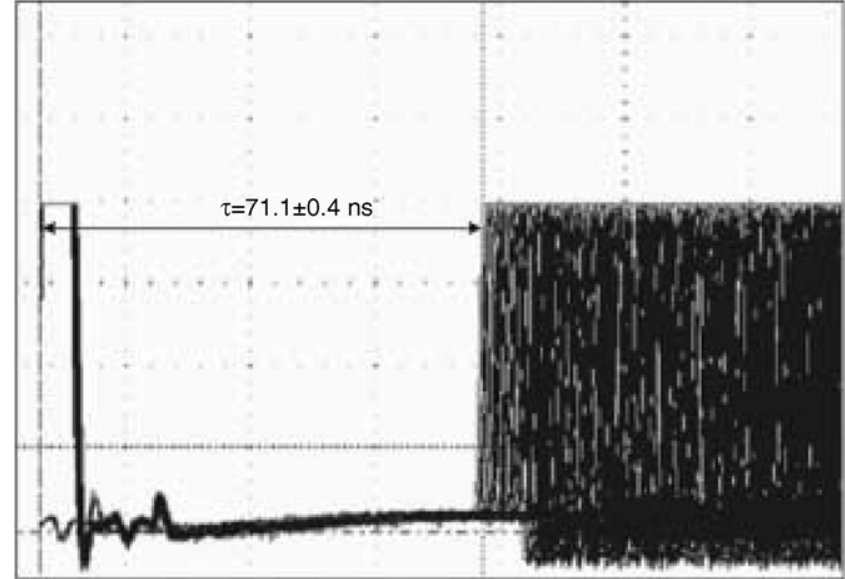

Fig. 1. Electronic dead time determination. The measured dead time is the interval from the leading edge of the trigger pulse to the next nearest pulse. The observed dead time from this specific measurement was $71.1 \pm 0.4 \mathrm{~ns}$.

results obtained by the Appelblad and Baxter approach are displayed, while the average of the dead-times evaluated according to Ramebäck et al. is inserted for comparison. There was never any significant discrepancy between these two ratio methods.

It has previously been reported that secondary electron multipliers suffers from apparently low reading due to loss of gain at high count-rates, i.e., sag. However, this effect is also reported to be more predominant in continuous dynode electron multipliers that in discrete dynode multipliers $[13,14]$. In the measurements performed in this study, count rates of up to $1.4 \times 10^{6} \mathrm{cps}$ for the major isotopes ${ }^{235} \mathrm{U}$ and ${ }^{238} \mathrm{U}$ were used and yet no significant effect of sagging could be found.

In Fig. 1 the result from a dead time determination with the electronic method is shown. The obvious advantage with this method is the precision with which the dead time can be determined. However, it is important to realize that, in this case, the electronically determined dead time is only that of the pulse amplifier of the detection system. The very first component in the signal chain, the electron multiplier, has its own dead time. Therefore, there is a series of components in the signal chain all having their own inherent dead time. Such series of dead times will be complicated to correct for [8], and Eq. (1) will not apply

Table 2

Results from the different dead time determinations

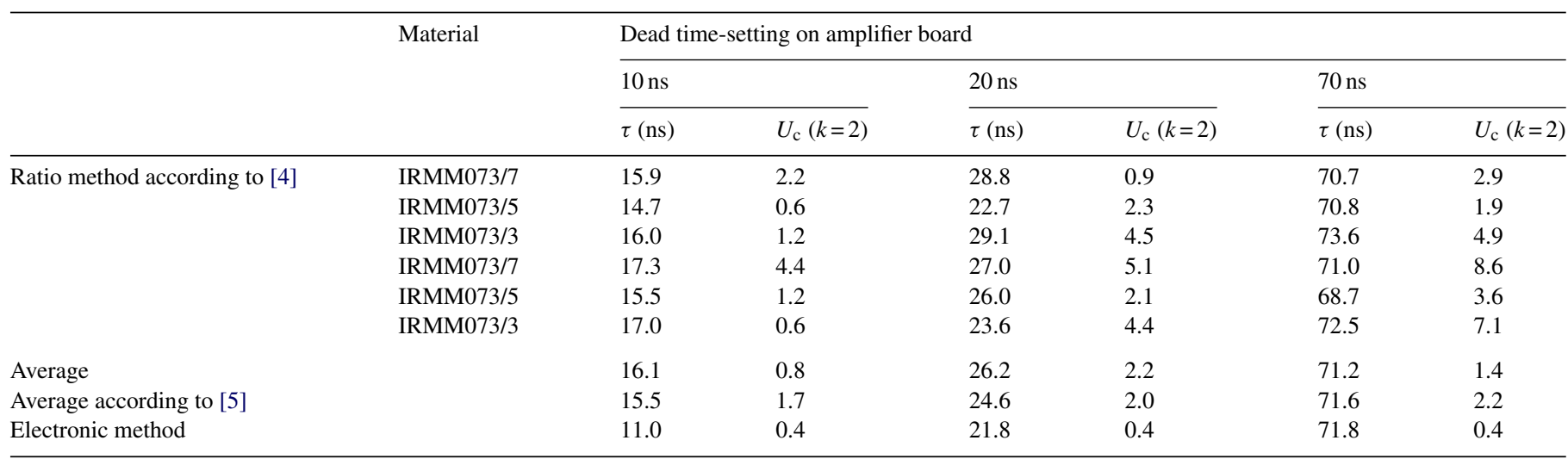




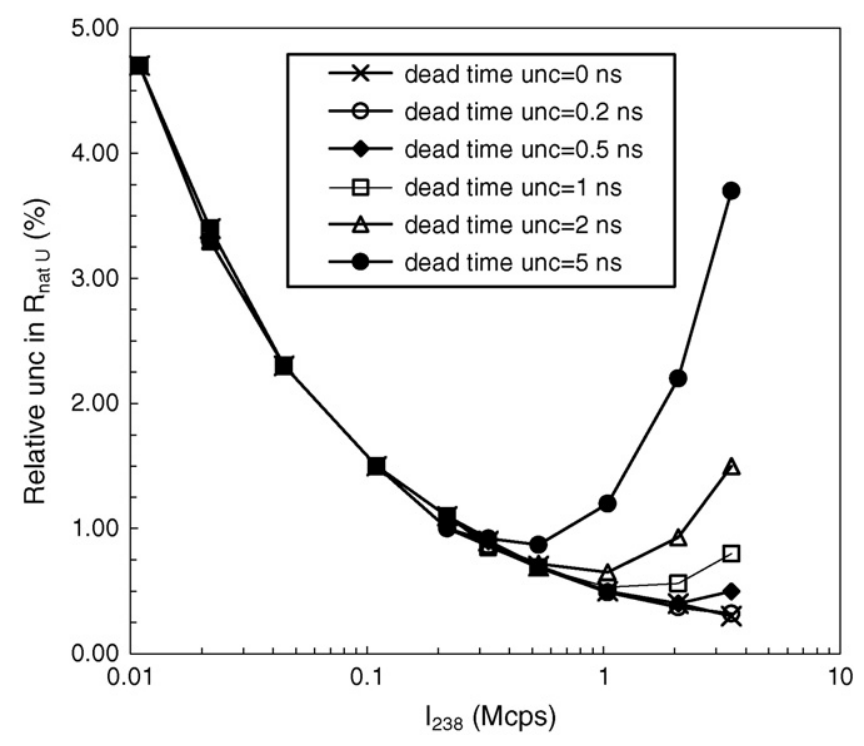

Fig. 2. The impact of dead time on the combined uncertainty in an observed isotope abundance ratio (natural uranium, $\tau=71.8 \mathrm{~ns}$ and a non-paralyzable dead time type). Observe that the uncertainty in the dead time is expressed for $k=1$, whereas $k=2$ is applied to the uncertainty of the isotope abundance ratio.

for such situations. However, if a sufficiently long dead time is introduced late in the signal chain, this component will effectively determine both the magnitude and type of dead time of the entire system [8]. As mentioned above, this seems to be the case for the longest dead time setting of the pulse amplifier.

Fig. 2 shows the calculated combined uncertainty in the determined natural uranium isotope abundance ratio for different dead time uncertainties $(k=1)$. A variable dead time will provide a significant contribution to the isotope abundance ratio uncertainty when pulse counting is used [15]. It can be seen in Fig. 2 that with the precision in electronic dead time determination achieved in this work, the contribution of the dead time to the combined isotope abundance ratio uncertainty is negligible. In fact, it only increases from 0.28 to $0.32 \%$ going from a zero dead time uncertainty to $\pm 0.4 \mathrm{~ns}$ at a count rate of $3.5 \mathrm{Mcps}$ on the major isotope. Ultimately, inclusion of the dead time contribution to a combined uncertainty calculation in mass spectrometry will therefore not be necessary.

Furthermore, the stability of an electronic dead time should be expected to be good, which was noticed by Schönfeld and Janssen [16]. Over a three year period of electronic measurements they observed only sub-nanosecond variability in the determined dead time. The same has been observed in our laboratory using sector field ICP-MS, which has shown no significant variations in the electronic dead time, $\tau=71.8 \pm 0.4 \mathrm{~ns}$, over the last two years. The dead time for continuous electron multipliers have been reported to vary both with the age of the detector
[17] and with the mass to charge ratio of the incoming ions [14]. The approach of implementing a relatively large, electronic dead time should minimize these effects as well.

\section{Conclusions}

We have shown that it is possible to configure the pulse detection system for an ICP-MS such that consistency between ratio and electronically determined dead times is achieved. Moreover, the study shows the importance of a correct dead time setting in a pulse counting system used in mass spectrometry in order to achieve a low uncertainty in the measured dead time. With electronic measurements, a dead time uncertainty of $\pm 0.4 \mathrm{~ns}$ was reached, which is about an order of magnitude better than can be obtained by ratio-based methods. More importantly this high level of precision will obviate the need to implement the otherwise considerable dead time contribution at high count rates in a complete uncertainty budget of isotope abundance ratio measurements.

\section{Acknowledgements}

Melker Nordstrand is gratefully acknowledged for the modification of the Element2 pulse amplifier, and Dr. Anna Sjögren for the preparation of the IRMM 073 solutions.

\section{References}

[1] J.M. Hayes, D.A. Schoeller, Anal. Chem. 49 (1977) 306.

[2] A.J. Fahey, Rev. Sci. Instrum. 69 (1998) 1282.

[3] A. Held, P.D.P. Taylor, J. Anal. At. Spectrom. 14 (1999) 1075.

[4] P.K. Appelblad, D.C. Baxter, J. Anal. At. Spectrom. 15 (2000) 557.

[5] H. Ramebäck, M. Berglund, D. Vendelbo, R. Wellum, P.D.P. Taylor, J. Anal. At. Spectrom. 16 (2001) 1271.

[6] S.M. Nelms, C.R. Quétel, T. Prohaska, J. Vogel, P.D.P. Taylor, J. Anal. At. Spectrom. 16 (2001) 333.

[7] J.W. Müller, Nucl. Instrum. Methods 112 (1973) 47.

[8] J.W. Müller, Nucl. Instrum. Methods A301 (1991) 543.

[9] G.F. Knoll, Radiation Detection and Measurement, Wiley, Chichester, 2000.

[10] J. Bochard, Appl. Radiat. Isot. 52 (2000) 441.

[11] A. Sjögren, P.K. Appelblad, A. Tovedal, H. Ramebäck, J. Anal. At. Spectrom. 20 (2005) 320.

[12] Guide to the Expression of Uncertainty in Measurements, International Organisation for Standardisation, ISO/GUM, Geneva, Switzerland, 1995, ISBN 92-67-10188-9.

[13] G.P. Russ III, J.M. Bazan, Spectrochim. Acta 42B (1987) 49

[14] F. Vanhaecke, G. de Wannemacker, L. Moens, R. Dams, C. Latkoczy, T. Prohaska, G. Stingeder, J. Anal. At. Spectrom. 13 (1998) 567.

[15] J. Diemer, C.R. Quétel, P.D.P. Taylor, J. Anal. At. Spectrom. 17 (2002) 1137.

[16] E. Schönfeld, H. Janssen, Nucl. Instrum. Methods A339 (1994) 137.

[17] A. Held, P.D.P. Taylor, J. Anal. At. Spectrom. 14 (1999) 1075. 